Proceedings of the Edinburgh Mathematical Society (2006) 49, 701-708 (C)

DOI:10.1017/S0013091504001142 Printed in the United Kingdom

\title{
A NOTE ON 2-LOCAL MAPS
}

\author{
YING-FEN LIN ${ }^{1}$ AND TSAI-LIEN WONG ${ }^{2}$ \\ ${ }^{1}$ Department of Mathematics Education, National Hualien University of Education, \\ Hua-Lien, Taiwan 970, Republic of China (linyf@mail.nhlue.edu.tw) \\ ${ }^{2}$ Department of Applied Mathematics, National Sun Yat-Sen University, \\ Kaohsiung, Taiwan 804, Republic of China (tlwong@math.nsysu.edu.tw)
}

(Received 13 October 2004)

\begin{abstract}
The aim of this note is to characterize 2-local automorphisms and derivations on matrix rings over finite-dimensional division rings.
\end{abstract}

Keywords: local automorphisms; local derivations; matrix rings

2000 Mathematics subject classification: Primary 16W20; 16W25; 15A33

\section{Introduction}

The study of local maps was initiated by Kadison [18] and Larson and Sourour [20]. In 1997, Šemrl [29] introduced the concepts of 2-local automorphisms and derivations on the algebra $B(H)$. Let $A$ be an algebra. A (non-additive) map $\varphi: A \rightarrow A$ is called a 2-local automorphism if, for every $a, b \in A$, there exists an automorphism $\sigma_{a, b}: A \rightarrow A$ such that $\varphi(a)=\sigma_{a, b}(a)$ and $\varphi(b)=\sigma_{a, b}(b)$. Similarly, a (non-additive) map $\delta: A \rightarrow A$ is called a 2-local derivation if, for every $a, b \in A$, there exists a derivation $d_{a, b}: A \rightarrow A$ such that $\delta(a)=d_{a, b}(a)$ and $\delta(b)=d_{a, b}(b)$.

Local and 2-local maps have been studied on different operator algebras by many authors $[\mathbf{2}-\mathbf{7}, \mathbf{1 5}-\mathbf{1 7}, \mathbf{1 9}, \mathbf{2 1}-\mathbf{2 8}]$.

It is interesting to note that the study of local maps on finite-dimensional algebras is sometimes more difficult than in the infinite-dimensional case. In [29], Šemrl described 2-local automorphisms on the algebra $B(H)$, all bounded linear operators on the infinitedimensional separable Hilbert space $H$. However, for the case when $H$ is finite dimensional, Šemrl's original proof was long and involved tedious computations. A similar description for the finite-dimensional case appeared later, in [19,24]. Our first goal is to describe 2-local automorphisms on matrix algebras over finite-dimensional division rings.

Theorem 1.1. Let $K$ be a finite-dimensional division algebra over its centre $Z$ with characteristic not 2 , and let $M_{n}(K), n \geqslant 1$, be the ring of $n \times n$ matrices over $K$. Then every 2-local automorphism of $M_{n}(K)$ is an automorphism or an anti-automorphism 
of $M_{n}(K)$. Moreover, if $n \geqslant 2$, then every 2-local automorphism of $M_{n}(K)$ is an automorphism of $M_{n}(K)$.

This result is a generalization of theorems due to Molnar [24] and Kim and Kim [19] obtained for $M_{n}(\mathbb{C})$. It also generalizes a theorem by Chebotar et al. [5, Theorem 5.3], where 2-local automorphisms of finite-dimensional division rings $K$ with characteristic 0 were described. It is interesting to note that the case of anti-automorphism (if $n=1$ ) is really possible (see [5, Example 5.4]).

Our second theorem gives a description of 2-local derivations on matrix algebras over finite-dimensional division rings.

Theorem 1.2. Let $K$ be a finite-dimensional division algebra over its centre $Z$ with characteristic not 2 , and let $M_{n}(K), n \geqslant 1$, be the ring of $n \times n$ matrices over $K$. Then every 2-local derivation of $M_{n}(K)$ is a derivation.

This result is a generalization of Kim and Kim's theorem [19] obtained for $M_{n}(\mathbb{C})$.

Finally, motivated by [5, Theorem 2.1], we prove the following result.

Theorem 1.3. Let $K$ be a division ring with centre $Z$ and let $M_{n}(K), n \geqslant 2$, be the ring of $n \times n$ matrices over $K$. Suppose that $\alpha: M_{n}(K) \rightarrow M_{n}(K)$ is a bijective additive map such that

$$
\alpha\left(a^{-1}\right) \alpha(a)=\alpha\left(b^{-1}\right) \alpha(b) \neq 0 \quad \text { for all invertible } a, b \in M_{n}(K) .
$$

Then $\alpha=\lambda \varphi$, where $\varphi: M_{n}(K) \rightarrow M_{n}(K)$ is an automorphism or an anti-automorphism and $\lambda=\alpha(1) \in Z$.

This result is connected with the well-known Hua theorem [14] and it generalizes some results of $[\mathbf{5}, \mathbf{1 0}]$.

\section{2-local automorphisms and derivations on matrix algebras over division rings}

Let $K$ be a finite-dimensional division algebra over its centre $Z$, and let $M_{n}(K)$ be the ring of $n \times n$ matrices over $K$. We denote by $e_{i j}$ the matrix unit, that is, the matrix which has a one in the $(i, j)$-position and zeros elsewhere.

Let $\operatorname{tr}: K \rightarrow Z$ be a reduced trace of $K$ and $\operatorname{Tr}: M_{n}(K) \rightarrow Z$ be the trace map of $M_{n}(K)$ defined by $\operatorname{Tr}(A)=\operatorname{tr}\left(a_{11}\right)+\operatorname{tr}\left(a_{22}\right)+\cdots+\operatorname{tr}\left(a_{n n}\right)$ if $A=\sum_{i, j} a_{i j} e_{i j} \in M_{n}(K)$.

We first recall the following result about the reduced trace (see, for example, $[\mathbf{9}$, p. 148, Lemma 4]).

Lemma 2.1. There exists an $a \in K$ such that $\operatorname{tr}(a) \neq 0$.

Lemma 2.2. If $A$ is non-zero in $M_{n}(K)$, then there exists a $B \in M_{n}(K)$ such that $\operatorname{Tr}(A B) \neq 0$.

Proof. We denote $A$ by $\sum_{i, j} a_{i j} e_{i j}$. Since $A \neq 0$, say $a_{s t} \neq 0$ in $K$ for some $1 \leqslant s, t \leqslant$ $n$. By Lemma 2.1, we can pick an $a \in K$ such that $\operatorname{tr}(a) \neq 0$. Let $B=a_{\text {st }}^{-1} a e_{t s}$. We have $A B=\sum_{i} a_{i t} a_{s t}^{-1} a e_{i s}$ and so $\operatorname{Tr}(A B)=\operatorname{tr}(a) \neq 0$ as desired. 
Now we can describe 2-local automorphisms of matrix algebras over finite-dimensional division rings using some ideas from $[\mathbf{5}, \mathbf{2 4}]$.

Proof of Theorem 1.1. Let $\varphi: M_{n}(K) \rightarrow M_{n}(K)$ be a 2-local automorphism. For every $x, y \in M_{n}(K)$, there exists an automorphism $\sigma_{x, y}$ on $M_{n}(K)$ such that $\varphi(x)=\sigma_{x, y}(x)$ and $\varphi(y)=\sigma_{x, y}(y)$. By [13, Theorem 4.3.1], there exists an invertible $c \in M_{n}(K)$ such that $\sigma_{x, y}(x)=c x c^{-1}$ and $\sigma_{x, y}(y)=c y c^{-1}$. Therefore,

$$
\varphi(x) \varphi(y)=\sigma_{x, y}(x) \sigma_{x, y}(y)=c x y c^{-1}
$$

and so

$$
\operatorname{Tr}(\varphi(x) \varphi(y))=\operatorname{Tr}(x y) \quad \text { for all } x, y \in M_{n}(K)
$$

Let $\left\{k_{1}, k_{2}, \ldots, k_{m}\right\}$ be a basis of $K$ over $Z$. We claim that $\varphi\left(k_{i} e_{j l}\right), 1 \leqslant i \leqslant m$, $1 \leqslant j, l \leqslant n$, are linearly independent over $Z$. Assume on the contrary that there exist $\lambda_{i j l}$ in $Z$ not all zero, say $\lambda_{i_{0} j_{0} l_{0}} \neq 0$, such that

$$
\sum_{i, j, l} \lambda_{i j l} \varphi\left(k_{i} e_{j l}\right)=0
$$

By Lemma 2.1, there exists an $a \in K$ such that $\operatorname{tr}(a) \neq 0$. Since $\sum_{i} \lambda_{i j_{0} l_{0}} k_{i} \neq 0$, let

$$
b=\left(\sum_{i} \lambda_{i j_{0} l_{0}} k_{i}\right)^{-1} a .
$$

It follows from (2.2) and the linearity of the trace map that

$$
\begin{aligned}
0 & =\operatorname{Tr}\left(\left[\sum_{i, j, l} \lambda_{i j l} \varphi\left(k_{i} e_{j l}\right)\right] \varphi\left(b e_{l_{0} j_{0}}\right)\right) \\
& =\sum_{i, j, l} \lambda_{i j l} \operatorname{Tr}\left(\varphi\left(k_{i} e_{j l}\right) \varphi\left(b e_{l_{0} j_{0}}\right)\right) \\
& =\sum_{i, j, l} \lambda_{i j l} \operatorname{Tr}\left(k_{i} b e_{j l} e_{l_{0} j_{0}}\right) \\
& =\sum_{i} \lambda_{i j_{0} l_{0}} \operatorname{tr}\left(k_{i} b\right) \\
& =\operatorname{tr}\left(\left(\sum_{i} \lambda_{i j_{0} l_{0}} k_{i}\right) b\right) \\
& =\operatorname{tr}(a),
\end{aligned}
$$

which is a contradiction. Therefore, the $\varphi\left(k_{i} e_{j l}\right), 1 \leqslant i \leqslant m, 1 \leqslant j, l \leqslant n$, are linearly independent over $Z$ and hence span $M_{n}(K)$ over $Z$. 
We can now prove the linearity of $\varphi$ over $Z$. For each $u, v \in M_{n}(K)$ and for every $i, j$, $l$, we find from (2.2) that

$$
\begin{aligned}
\operatorname{Tr}\left(\varphi(u+v) \varphi\left(k_{i} e_{j l}\right)\right) & =\operatorname{Tr}\left((u+v) k_{i} e_{j l}\right) \\
& =\operatorname{Tr}\left(u k_{i} e_{j l}\right)+\operatorname{Tr}\left(v k_{i} e_{j l}\right) \\
& =\operatorname{Tr}\left(\varphi(u) \varphi\left(k_{i} e_{j l}\right)\right)+\operatorname{Tr}\left(\varphi(v) \varphi\left(k_{i} e_{j l}\right)\right) \\
& =\operatorname{Tr}\left((\varphi(u)+\varphi(v)) \varphi\left(k_{i} e_{j l}\right)\right) .
\end{aligned}
$$

Since the $\varphi\left(k_{i} e_{j l}\right)$ span $M_{n}(K)$ over $Z$, we have

$$
\operatorname{Tr}((\varphi(u+v)-\varphi(u)-\varphi(v)) x)=0 \quad \text { for all } x, u, v \in M_{n}(K) .
$$

By Lemma 2.2, we have $\varphi(u+v)-\varphi(u)-\varphi(v)=0$. That is, $\varphi(u+v)=\varphi(u)+\varphi(v)$ for all $u, v \in M_{n}(K)$.

For each $\alpha \in Z$ and $u \in M_{n}(K)$, there exists an automorphism $\sigma_{u, \alpha u}$ such that $\varphi(u)=\sigma_{u, \alpha u}(u)$ and $\varphi(\alpha u)=\sigma_{u, \alpha u}(\alpha u)$. Then

$$
\varphi(\alpha u)=\sigma_{u, \alpha u}(\alpha u)=\alpha \sigma_{u, \alpha u}(u)=\alpha \varphi(u) .
$$

That is, $\varphi$ is a linear map on $M_{n}(K)$ over $Z$. Being a 2-local automorphism, $\varphi$ is injective and hence is surjective, since $M_{n}(K)$ is finite dimensional over $Z$.

Note that, for each $u \in M_{n}(K)$, there exists an automorphism $\sigma_{u, u^{2}}$ such that $\varphi(u)=$ $\sigma_{u, u^{2}}(u)$ and $\varphi\left(u^{2}\right)=\sigma_{u, u^{2}}\left(u^{2}\right)$. Then $\varphi\left(u^{2}\right)=\sigma_{u, u^{2}}\left(u^{2}\right)=\sigma_{u, u^{2}}(u)^{2}=\varphi(u)^{2}$ for all $u \in M_{n}(K)$. Therefore, $\varphi$ is a Jordan automorphism. Since the characteristic of $K$ is not 2, it follows from the Herstein theorem [11] that $\varphi$ is an automorphism or an antiautomorphism.

Finally, let $n>1$. Suppose that $\varphi$ is an anti-automorphism. Substituting $x=e_{11}$ and $y=e_{12}$ in $(2.1)$, we obtain $0=\varphi(y x)=\varphi(x) \varphi(y)=c x y c^{-1}$, which is a contradiction.

We shall now describe 2-local derivations of matrix algebras over finite-dimensional division rings.

Proof of Theorem 1.2. Let $\delta: M_{n}(K) \rightarrow M_{n}(K)$ be a 2-local derivation. For each $x, y \in M_{n}(K)$, there exists a derivation $d_{x, y}$ on $M_{n}(K)$ such that $\delta(x)=d_{x, y}(x)$ and $\delta(y)=d_{x, y}(y)$. By the proposition in [13, p. 100], there exists an invertible $c \in M_{n}(K)$ such that $[c, x y]=d_{x, y}(x y)=d_{x, y}(x) y+x d_{x, y}(y)=\delta(x) y+x \delta(y)$. Thus, we have

$$
0=\operatorname{Tr}([c, x y])=\operatorname{Tr}(\delta(x) y+x \delta(y)) \quad \text { and so } \quad \operatorname{Tr}(\delta(x) y)=-\operatorname{Tr}(x \delta(y)) .
$$

Therefore,

$$
\begin{aligned}
\operatorname{Tr}(\delta(u+v) z) & =-\operatorname{Tr}((u+v) \delta(z)) \\
& =-\operatorname{Tr}(u \delta(z))-\operatorname{Tr}(v \delta(z)) \\
& =\operatorname{Tr}(\delta(u) z)+\operatorname{Tr}(\delta(v) z) \\
& =\operatorname{Tr}((\delta(u)+\delta(v)) z)
\end{aligned}
$$


and so

$$
\operatorname{Tr}((\delta(u+v)-\delta(u)-\delta(v)) z)=0 \quad \text { for all } u, v, z \in M_{n}(K) .
$$

By Lemma 2.2, we have $\delta(u+v)-\delta(u)-\delta(v)=0$. That is, $\delta(u+v)=\delta(u)+\delta(v)$ for all $u, v \in M_{n}(K)$.

Finally, for each $u \in M_{n}(K)$, there exists a derivation $d_{u, u^{2}}$ such that $\delta(u)=d_{u, u^{2}}(u)$ and $\delta\left(u^{2}\right)=d_{u, u^{2}}\left(u^{2}\right)$. Then

$$
\delta\left(u^{2}\right)=d_{u, u^{2}}\left(u^{2}\right)=d_{u, u^{2}}(u) u+u d_{u, u^{2}}(u)=\delta(u) u+u \delta(u) \text { for all } u \in M_{n}(K) .
$$

Therefore, $\delta$ is a Jordan derivation. Since the characteristic of $K$ is not 2, we see that $\delta$ is a derivation by the Herstein theorem [12].

\section{A generalization of Hua's theorem}

In 1949, Hua [14] proved that every bijective additive map $\alpha$ on a division $\operatorname{ring} K$ satisfying $\alpha(a b a)=\alpha(a) \alpha(b) \alpha(a)$ and $\alpha(1)=1$ is an automorphism or an anti-automorphism. This result was reformulated by Artin as: any bijective additive map $\alpha$ on a division ring $K$ satisfying $\alpha\left(a^{-1}\right)=\alpha(a)^{-1}$ and $\alpha(1)=1$ is an automorphism or an antiautomorphism [1, Theorem 1.15]. The same result was established for the $n \times n$ matrix rings over a division ring $K$ in case when $K \neq \mathrm{GF}(2)$, the Galois field of two elements [10]. In [5], the authors removed the condition of $\alpha(1)=1$ in Hua's result and prove the following.

Theorem 3.1 (Chebotar et al. [5, Theorem 2.1]). Let $K$ be a division ring with centre $Z$ and $\alpha: K \rightarrow K$ be a bijective additive map such that

$$
\alpha\left(a^{-1}\right) \alpha(a)=\alpha\left(b^{-1}\right) \alpha(b) \quad \text { for all non-zero } a, b \in K .
$$

Then $\alpha=\lambda \varphi$, where $\varphi: K \rightarrow K$ is an automorphism or an anti-automorphism and $\lambda=\alpha(1) \in Z$.

We shall generalize this result to matrix algebras over division rings. We begin with some technical results.

Lemma 3.2. Let $K$ be a division ring with centre $Z$ such that $K \neq \mathrm{GF}(2)$ and let $M_{n}(K), n \geqslant 2$, be the ring of $n \times n$ matrices over $K$. Suppose that $\alpha: M_{n}(K) \rightarrow$ $M_{n}(K)$ is a surjective additive map. If $\mu \in M_{n}(K)$ satisfies $[\mu, \alpha(y)]=0$ for all invertible $y \in M_{n}(K)$ with $y-1$ invertible, then $\mu \in Z$.

Proof. We claim first that $\left[\mu, \alpha\left(k e_{i j}\right)\right]=0$ for all $k \in K$ and $1 \leqslant i, j \leqslant n$. If $k=$ 0 , then the above equality holds automatically. Let $0 \neq k \in K$ and $1 \leqslant i, j \leqslant n$. In the case when $i \neq j$, we pick $h \in K$ such that $h \neq 0,1$. Let $y_{1}=h+k e_{i j}$ and $y_{2}=h$; we find that $y_{l}$ and $y_{l}-1$ are invertible and so $\left[\mu, \alpha\left(y_{l}\right)\right]=0$ for $l=1,2$. Therefore, $\left[\mu, \alpha\left(k e_{i j}\right)\right]=\left[\mu, \alpha\left(y_{1}\right)-\alpha\left(y_{2}\right)\right]=0$. In the case when $i=j$, we consider $y=$ $k e_{i i}+e_{12}+e_{23}+\cdots e_{n-1 n}+e_{n 1}$. Since $y$ and $y-1$ are invertible, we have $[\mu, \alpha(y)]=0$. It follows from the above case that

$$
0=\left[\mu, \alpha\left(e_{12}\right)\right]=\left[\mu, \alpha\left(e_{23}\right)\right]=\cdots=\left[\mu, \alpha\left(e_{n-1 n}\right)\right]=\left[\mu, \alpha\left(e_{n 1}\right)\right],
$$


and so $\left[\mu, \alpha\left(k e_{i i}\right)\right]=0$. Since $\alpha$ is a surjective additive map, by the claim, we have $\mu \in Z$ as desired.

Our next goal is the case when $K=\mathrm{GF}(2)$.

Lemma 3.3. Suppose $K=\mathrm{GF}(2)$ and $n \geqslant 2$. Let $\alpha$ be a surjective additive map of $M_{n}(K)$ and $\mu \in M_{n}(K)$.

(i) If $\mu$ satisfies $[\mu, y]=0$ for all invertible $y \in M_{n}(K)$, then $\mu \in K$.

(ii) If $\mu$ satisfies $[\mu, \alpha(y)]=0$ for all invertible $y \in M_{n}(K)$, then $\mu \in K$.

Proof. (i) Let $i \neq j$. By assumption, we have $[\mu, 1]=0$ and $\left[\mu, 1+e_{i j}\right]=0$, and therefore $\left[\mu, e_{i j}\right]=0$. Further, since

$$
\left[\mu, e_{i i}+e_{i j}+e_{j i}+\sum_{k \neq i, j} e_{k k}\right]=0 \text { and }\left[\mu, e_{i j}+e_{j i}+\sum_{k \neq i, j} e_{k k}\right]=0
$$

it follows that $\left[\mu, e_{i i}\right]=0$. Hence, $\mu \in K$ as desired.

(ii) Since $\alpha$ is additive, we can see from the above proof that $\left[\mu, \alpha\left(e_{i j}\right)\right]=0$ for all $1 \leqslant i, j \leqslant n$. From the fact that $\alpha$ is surjective and additive, it follows that $\mu \in K$.

Proof of Theorem 1.3. Let $z=\alpha\left(1^{-1}\right) \alpha(1) \neq 0$; then $z=\alpha\left(a^{-1}\right) \alpha(a)=\alpha(a) \alpha\left(a^{-1}\right)$ and so

$$
\alpha(a) z=\alpha(a)\left(\alpha\left(a^{-1}\right) \alpha(a)\right)=\left(\alpha(a) \alpha\left(a^{-1}\right)\right) \alpha(a)=z \alpha(a)
$$

for all invertible $a \in M_{n}(K)$. By Lemmas 3.2 and 3.3(ii), we have $z \in Z$.

Suppose first that $K \neq \mathrm{GF}(2)$. Let $\lambda=\alpha(1)$ and let $\varphi: M_{n}(K) \rightarrow M_{n}(K)$ be defined by $\varphi(a)=\lambda^{-1} \alpha(a)$ for all $a \in M_{n}(K)$. Then $\varphi$ is a bijective additive map on $M_{n}(K)$ with $\varphi(1)=1$. If we can claim $\lambda \in Z$, then we will have $\varphi\left(a^{-1}\right) \varphi(a)=$ $z^{-1} \alpha\left(a^{-1}\right) \alpha(a)=z^{-1} z=1$ for all invertible $a \in M_{n}(K)$. Hence, $\varphi$ is an automorphism or an anti-automorphism in light of [10] and so the proof will be complete.

Let $x, y \in M_{n}(K)$ be invertible elements such that $x-y^{-1}$ is invertible. Thus, we have the following beautiful identity due to Hua:

$$
\left(x^{-1}-\left(x-y^{-1}\right)^{-1}\right)^{-1}=x-x y x .
$$

Set $x=1$ and let $y$ be an invertible element such that $y-1$ is invertible (and hence $1-y^{-1}=y^{-1}(y-1)$ is invertible). Applying $\alpha$ to (3.1) and using $\alpha\left(a^{-1}\right)=z \alpha(a)^{-1}$, we 
obtain

$$
\begin{aligned}
\alpha(y) & =\lambda-\alpha\left(\left(1-\left(1-y^{-1}\right)^{-1}\right)^{-1}\right) \\
& =\lambda-z \alpha\left(1-\left(1-y^{-1}\right)^{-1}\right)^{-1} \\
& =\lambda-z\left(\lambda-\alpha\left(\left(1-y^{-1}\right)^{-1}\right)\right)^{-1} \\
& =\lambda-z\left(\lambda-z\left(\lambda-\alpha\left(y^{-1}\right)\right)^{-1}\right)^{-1} \\
& =\lambda-\left(\lambda^{-1}-\left(\lambda-z \alpha(y)^{-1}\right)^{-1}\right)^{-1} \\
& =\lambda-\left(\lambda^{-1}-\left(\lambda-\left(z^{-1} \alpha(y)\right)^{-1}\right)^{-1}\right)^{-1} \\
& =\lambda-\left(\lambda-\lambda^{-1} \alpha(y) \lambda\right) \\
& =\lambda^{-1} \alpha(y) \lambda .
\end{aligned}
$$

Hence, $[\lambda, \alpha(y)]=0$ for all invertible $y \in M_{n}(K)$ with $y-1$ invertible. By Lemma 3.2, we have $\lambda \in Z$ as desired.

Suppose next that $K=Z=\mathrm{GF}(2)$. Let $a$ be an invertible element of $M_{n}(K)$. It follows from $0 \neq z=\alpha(a) \alpha\left(a^{-1}\right) \in K$ that $\alpha(a)$ is invertible. Therefore, $\alpha$ is an invertibilitypreserving map. Since $\alpha$ is a bijective map on the finite set $M_{n}(\mathrm{GF}(2))$, it maps singular matrices to singular matrices. It follows from Dieudonné's [8] result that $\alpha$ must have the form of $\alpha(X)=U X V$ or $\alpha(X)=U X^{t} V$, where $U, V \in M_{n}(K)$ are invertible and $t$ is the transpose map.

Say $\alpha(X)=U X V$. Let $a$ be an invertible element in $M_{n}(K)$. It follows from

$$
\alpha\left(a^{-1}\right) \alpha(a)=\alpha(1)^{2}
$$

that $U a^{-1} V U a V=U V U V$, i.e. $[V U, a]=0$ for all invertible $a$. Therefore, $V U \in K$ by Lemma 3.3(i) and so $U V=V U$. Hence, we have $\alpha(1)=U V=V U \in K$ and $\alpha(X)=U X V=U V\left(V^{-1} X V\right)=\alpha(1)\left(V^{-1} X V\right)$ as desired. Similar arguments can be applied for the case $\alpha(X)=U X^{t} V$. The proof is completed.

Acknowledgements. T.-L.W. was supported in part by the National Science Council under grant no. NSC92-2115-M-110-010.

\section{References}

1. E. ARTin, Geometric algebra (Interscience, New York, 1957).

2. M. BARCZY AND M. Tóth, Local automorphisms of the sets of states and effects on a Hilbert space, Rep. Math. Phys. 48 (2001), 289-298.

3. M. BREŠAR AND P. ŠEMRL, Mappings which preserve idempotents, local automorphisms, and local derivations, Can. J. Math. 45 (1993), 483-496.

4. M. BREŠAR AND P. ŠEMRL, On local automorphisms and mappings that preserve idempotents, Studia Math. 113 (1995), 101-108.

5. M. A. Chebotar, W.-F. Ke, P.-H. Lee and L.-S. Shiao, On maps preserving products, Can. Math. Bull. 48 (2005), 355-369.

6. R. CRIST, Local derivations on operator algebras, J. Funct. Analysis 135 (1996), 76-92.

7. R. CRIST, Local automorphisms, Proc. Am. Math. Soc. 128 (2000), 1409-1414.

8. J. Dieudonné, Sur une generalisation du groupe orthogonal à quatre variables, Arch. Math. 1 (1949), 282-287. 
9. P. K. Draxl, Skew fields, London Mathematical Society Lecture Note Series, Volume 81 (Cambridge University Press, 1983).

10. H. Essannouni AND A. KAIDi, Le théorème de Hua pour les algèbres artiniennes simples, Linear Alg. Applic. 297 (1999), 9-22.

11. I. N. Herstein, Jordan homomorphisms, Trans. Am. Math. Soc. 81 (1956), 331-351.

12. I. N. Herstein, Jordan derivations of prime rings, Proc. Am. Math. Soc. 8 (1957), 11041110 .

13. I. N. Herstein, Noncommutative rings, Carus Mathematical Monographs, Volume 15 (Wiley, 1968).

14. L.-K. HuA, On the automorphisms of a field, Proc. Natl Acad. Sci. USA 35 (1949), 386-389.

15. W. Jing, Local derivations of reflexive algebras, Proc. Am. Math. Soc. 125 (1997), 869873.

16. W. JING, Local derivations of reflexive algebras, II, Proc. Am. Math. Soc. 129 (2001), 1733-1737.

17. W. JING, S. LU AND P. LI, Characterization of derivations on some operator algebras, Bull. Austral. Math. Soc. 66 (2002), 227-232.

18. R. V. Kadison, Local derivations, J. Alg. 130 (1990), 494-509.

19. S. O. Kim And J. S. Kim, Local automorphisms and derivations on $M_{n}$, Proc. Am. Math. Soc. 132 (2004), 1389-1392.

20. D. LARson AND A. Sourour, Local derivations and local automorphisms of $B(X)$, in Operator Theory: Operator Algebras and Applications, Part 2, Durham, NH, 1988, Proceedings of Symposia in Pure Mathematics, Volume 51, Part 2, pp. 187-194 (American Mathematical Society, Providence, RI, 1990).

21. L. Molnar, Local automorphisms of some quantum mechanical structures, Lett. Math. Phys. 58 (2001), 91-100.

22. L. Molnar, Some characterizations of the automorphisms of $B(H)$ and $C(X)$, Proc. Am. Math. Soc. 130 (2002), 111-120.

23. L. Molnar, 2-local isometries of some operator algebras, Proc. Edinb. Math. Soc. 45 (2002), 349-352.

24. L. Molnar, Local automorphisms of operator algebras on Banach spaces, Proc. Am. Math. Soc. 131 (2003), 1867-1874.

25. L. Molnar And P. SEMrL, Local automorphisms of the unitary group and the general linear group on a Hilbert space, Expo. Math. 18 (2000), 231-238.

26. L. MOLNAR AND B. ZALAR, On local automorphisms of group algebras of compact groups, Proc. Am. Math. Soc. 128 (2000), 93-99.

27. T. Petek And P. ŠEmrl, Adjacency preserving maps on matrices and operators, Proc. R. Soc. Edinb. A 132 (2002), 661-684.

28. E. Scholz AND W. Timmermann, Local derivations, automorphisms and commutativity preserving maps on $L^{+}(D)$, Publ. RIMS Kyoto 29 (1993), 977-995.

29. P. ŠEmrL, Local automorphisms and derivations on $B(H)$, Proc. Am. Math. Soc. 125 (1997), 2677-2680. 